\title{
Effects of live cells of Saccharomyces cerevisiae on in vitro lactate utilization by Megasphaera elsdenii and on the interactions between Streptococcus bovis and $M$. elsdenii grown on glucose
}

\author{
F Chaucheyras 1,2, G Fonty 1, G Bertin 2, Ph Gouet 1 \\ IINRA, Laboratoire de Microbiologie, C.R. de Clermont-Ferrand-Theix \\ 63122 Saint - Genes - Champanelle,; ${ }^{2}$ Santel - groupe Agritek, 85 rue Anatole France, \\ 92300 Levallois - Perret, France
}

Feeding high concentrate diets to ruminants may be detrimental to the ruminal digestion efficiency. The fermentation of readily degradable carbohydrates leads to lactic acid production in the rumen. Lactic acid is produced by several fermentative microbial species such as Streptococcus bovis. When the concentrate level is high, lactic acid, which is normally metabolized by bacterial species such as Megasphaera elsdenii, may accumulate and cause a great decline in ruminal $\mathrm{pH}$, that consequently induces digestive disorders. The positive effect of several microbial additives such as Aspergillus oryzae on in vitro lactate utilization by Selenomonas ruminantium (Nisbet and Martin, 1993, Curr Microbiol, 26, 133-136) and Megasphaera elsdenii (Waldrip and Martin, 1993, J Anim Sci, 71, 2770-2776) and also the efficiency of live yeasts on the stabilization of ruminal $\mathrm{pH}$ and other digestive parameters in vivo (Williams et al, 1991, J Anim Sci, 69, 3016-3026) have recently been shown. Nevertheless, the mechanisms of action of these additives remain unclear. The aim of this study was to find out the effects and the mechanisms of action of a live strain of Saccharomyces cerevisiae (CNCM 1-1077,
Institut Pasteur, France) on lactate utilization by a strain of Megasphaera elsdenii, and on the interactions between Streptococcus bovis, a lactate producer, and $M$. elsdenii, grown on glucose in vitro.

The addition of yeast cells increased the incorporation of L-lactate in whole cells of $M$. elsdenii. The stimulatory effect correlated with the concentration of yeast cells added, but was similar whether the yeast were alive or dead. Amino acids and vitamins seemed to be implicated in the stimulation. In a coculture of $M$. elsdenii and S. bovis on glucose, the addition of live yeasts led to a diminution of lactate production by $S$. bovis. At the beginning of the incubation, there was competition for glucose utilization between the yeast strain and the bacterial species, leading to a great decline of lactate production. In a second stage, the efficiency of $M$. elsdenii for lactate metabolism was reinforced by the presence of yeast cells.

These results demonstrate that live yeasts can be an interesting tool for the reduction of lactic acid production and also enhance its elimination from the rumen. Yeasts could help to limit the risks of acidosis when diets are rich in rapidly fermentable carbohydrates.

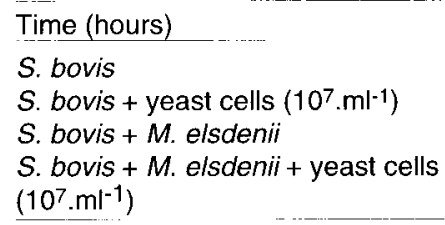

\begin{tabular}{cccc}
\multicolumn{4}{c}{ Lactate $(\mathrm{mM})$} \\
2 & 4 & 6 & 8 \\
$14.5 \pm 0.4$ & $14.8 \pm 0.1$ & $14.0 \pm 0.9$ & $14.6 \pm 0.3$ \\
$6.7 \pm 0.8$ & $7.7 \pm 0.1$ & $7.7 \pm 0.3$ & $7.8 \pm 0.4$ \\
$9.7 \pm 0.05$ & $7.7 \pm 0.3$ & $5.6 \pm 0.05$ & $3.5 \pm 0.03$ \\
$6.4 \pm 0.1$ & $4.5 \pm 0.4$ & $2.4 \pm 0.5$ & $1.6 \pm 0.1$
\end{tabular}

